Canadian

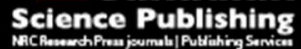

Canadian Journal of Physiology and Pharmacology Revue canadienne de physiologie et pharmacologie

\title{
EPAC activation inhibits acetaldehyde-induced activation and proliferation of hepatic stellate cell via Rap1
}

\begin{tabular}{|r|l|}
\hline Journal: & Canadian Journal of Physiology and Pharmacology \\
\hline Manuscript ID & cjpp-2015-0437.R1 \\
\hline Manuscript Type: & Article \\
\hline Date Submitted by the Author: & 20-Oct-2015 \\
\hline Complete List of Authors: & $\begin{array}{l}\text { Yang, Yan; Anhui Medical University } \\
\text { Yang, Feng; Anhui Medical University } \\
\text { Wu, Xiaojuan; Anhui Medical University } \\
\text { Lv, Xiongwen; Anhui Medical University } \\
\text { Li, Jun; Anhui Medical University }\end{array}$ \\
\hline Keyword: & acetaldehyde, hepatic stellate cells, EPAC1, EPAC2, Rap1 \\
\hline \multicolumn{2}{|l}{} \\
\hline
\end{tabular}

SCHOLARONE

Manuscripts 


\title{
EPAC activation inhibits acetaldehyde-induced activation and proliferation of hepatic stellate cell via Rap1
}

\author{
Yan Yang ${ }^{\mathrm{a}, \mathrm{b}}$, Feng Yang ${ }^{\mathrm{a}, \mathrm{b}}$, Xiaojuan $\mathrm{Wu}^{\mathrm{a}, \mathrm{b}}$, Xiongwen $\mathrm{Lv}^{\mathrm{a}, \mathrm{b}, *}, \mathrm{Jun} \mathrm{Li}^{\mathrm{a}, \mathrm{b}}$ \\ ${ }^{a}$ School of Pharmacy, Anhui Medical University, Meishan Road, Hefei, Anhui Province, 230032, China \\ ${ }^{\mathrm{b}}$ Institute for Liver Disease of Anhui Medical University, Meishan Road, Hefei, Anhui Province, 230032, China \\ Meishan Road, Hefei, Anhui Province, Hefei, 230032, China. \\ 1xw20150812@aliyun.com
}




\begin{abstract}
Hepatic stellate cells (HSCs) activation represents an essential event during alcoholic liver fibrosis (ALF). Previous studies have demonstrated that the rat HSCs could be significantly activated after exposure to $200 \mu \mathrm{M}$ acetaldehyde for $48 \mathrm{~h}$, and the cAMP/PKA signaling pathways were also dramatically upregulated in activated HSCs isolated from alcoholic fibrotic rat liver. Exchange protein activated by cAMP (EPAC) is a family of guanine nucleotide exchange factors (GEFs) for the small Ras-like GTPases Rap, and is being considered as a vital mediator of cAMP signaling in parallel with the principal cAMP target protein kinase A (PKA). Our data showed that both cAMP/PKA and cAMP/EPAC signaling pathways were involved in acetaldehyde-induced HSCs. Acetaldehyde could reduce the expression of EPAC1 while enhancing the expression of EPAC2. The cAMP analog Me-cAMP, which stimulates the EPAC/Rap1 pathway, could significantly decrease the proliferation and collagen synthesis of acetaldehyde-induced HSCs. Furthermore, depletion of EPAC2, but not EPAC1, prevented the activation of HSC measured as the production of $\alpha$-SMA and collagen type I and III, indicating that EPAC1 appears to have protective effects on acetaldehyde-induced HSCs. Curiously, activation of PKA or EPAC perhaps has opposite effects on the synthesis of collagen and $\alpha$-SMA, EPAC activation by Me-cAMP increased the levels of GTP-bound (activated) Rap1 while PKA activation by Phe-cAMP had no significant effects on such binding. These results suggested that EPAC activation could inhibit the activation and proliferation of acetaldehyde-induced HSCs via Rap1.
\end{abstract}

Keywords: acetaldehyde EPAC1 EPAC2 hepatic stellate cells Rap1 


\section{Introduction}

About 3.3 million deaths (5.9\% of all deaths) were attributed to alcohol consumption in 2012 according to the 2014 WHO Global Report (Collart et al. 2015). Alcoholic liver disease (ALD) is one of the most common etiologies of liver disease and has become a main cause of morbidity and mortality worldwide (Lachenmeier et al. 2014; Ramaiah et al. 2004). ALD encompasses a broad spectrum of disorders(,) ranging from fatty liver to more severe forms of liver injury, including alcoholic hepatitis, fibrosis, cirrhosis, and superimposed hepatocellular carcinoma (Gao and Bataller 2011; Orman et al. 2013). Alcoholic liver fibrosis (ALF) is identified as a breaking point in ALD because it can lead to cirrhosis (Duddempudi 2012). Therefore, the research on the molecular pathways and therapeutic strategies for ALF has attracted considerable attention in scholarly circles.

Although the development of ALF is complicated involving different molecular and biological mechanisms, mounting evidence suggests that the principal metabolite of ethanol, acetaldehyde, plays a critical role in the onset and maintenance of the fibrogenetic process. Many studies have demonstrated that acetaldehyde stimulates the deposition of extracellular matrix (ECM) by activating the hepatic stellate cells (HSCs), the major cell type involved in ALF (Friedman 1999; Hernandez-Gea and Friedman 2011; Mello et al. 2008). As response to acetaldehyde-induced stimulation, HSCs change from quiescent vitamin A-storing cells to activated myofibroblast-like cells, which proliferate and become the primary source of ECM (Luo et al. 2013).

The cyclic adenosine $3^{\prime}, 5^{\prime}$-monophosphate (cAMP) is a prominent multifunctional intracellular second messenger (de Rooij et al. 1998). By acting as a master regulator of innate immune cell function, increases in intracellular cAMP generally down-regulate inflammatory mediator generation, phagocytosis, and microbial killing (Serezani et al. 2008). Ethanol has been shown to be a well-known developmental toxicant with immunomodulatory properties (Tonk et al. 2013). Impaired immunity has also been observed in animal models of ethanol exposure. The liver is an organ with predominant innate immunity, and ethanol-induced dysregulation of innate immunity contributes to the pathogenesis of ALD. Toll-like receptors (TLRs) are a class of proteins that play a key role in the innate immune system. Recent studies have shown that the activation of TLR4 signaling in HSCs could promote liver fibrosis during alcohol consumption as evidenced in innate 
immunity in ALD (Gao et al. 2011). In addition, the spleen is also thought to be central in regulating the immune system with regard to non-alcoholic fatty liver disease (NAFLD), and previous research has shown that splenomegaly was secondary to portal hypertension with associated liver cirrhosis (Tarantino 2011). Gualde and Harizi have previously reported that splenic macrophages, dendritic cells, and lymphocytes contribute to prostaglandin E2 (PGE2) synthesis in the spleen (Gualde and Harizi 2004). Katia et al. have also found that PGE2 regulate Th17 cell differentiation and function through cAMP and EP2/EP4 receptor signaling (Boniface et al. 2009). These results further suggest that a better understanding of the cAMP axis is likely to provide new insights into the regulation of innate immunity, which would provide therapeutic benefit for ALD.

Most of the effects of cAMP are dependent upon the activation of its downstream effectors protein kinase A (PKA). In our previous study, we found that the activation of cAMP/PKA signaling pathway promotes the activation and proliferation of HSCs (Wang et al. 2014; Wang et al. 2015; Yang et al. 2015). cAMP acts via three mechanisms: PKA, Exchange protein activated by cAMP (EPAC) and cyclic nucleotide-gated (CNG) ion channels (Insel et al. 2012; Roberts and Dart 2014). Interestingly, it has recently been shown that EPAC mediate the antifibrotic effect of cAMP (Yokoyama et al. 2008b). EPAC proteins regulate many cellular processes, including migration, proliferation and apoptosis, via a PKA-independent pathway (Okumura et al. 2014; Yokoyama et al. 2008a). There are two main EPAC isoforms (EPAC1 and EPAC2, also known as RapGEF3 and RapGEF4, respectively) that are expressed from different genes in a variety of tissues (de Rooij et al. 1998; Roscioni et al. 2008; Ueno et al. 2001). EPAC proteins respond to cAMP and act as specific guanine nucleotide exchange factors (GEFs) for the small G proteins, Rap1 and Rap2 which were activated by exchanging bound GDP for GTP, while GTPase activating proteins return them to inactive GDP-bound states (Breckler et al. 2011; de Rooij et al. 1998; Jeyaraj et al. 2011; Schmidt et al. 2013). Based on these findings, we hypothesized that cAMP may influence acetaldehyde-induced HSCs activation by regulating the activation of both PKA and EPAC signaling pathways.

In this study, the rat HSC line were cultured and treated with $200 \mu \mathrm{M}$ acetaldehyde for $48 \mathrm{~h}$, which was proposed to mimic the activation process in vivo. We then tried to identify the role of cAMP/EPAC/Rap1 signaling pathway in the regulation of HSCs activation and proliferation induced by acetaldehyde. 


\section{Materials and methods}

\section{Antibodies and reagents}

Acetaldehyde (40\%) was purchased from Tianjin DaMao Chemical Reagent (Tianjin, China); 8-(4-Chlorophenylthio)-2'-O-methyladenosine 3',5'-cyclic monophosphate monosodium hydrate (Me-cAMP, also known as 007) and N6-phenyladenosine-3,5-cyclic monophosphate (Phe-cAMP) were obtained from Sigma-Aldrich (Lee et al.); $\alpha$-Smooth Muscle Actin ( $\alpha$-SMA) Antibody was purchased from Abcam (Lee et al.); Antibodies for Collagen type I, Collagen type III, were purchased from Santa Cruz (CA, USA); Antibodies for EPAC1, EPAC2 and Beta-Actin ( $\beta$-actin) were obtained from Biosynthsis (Beijing, China); secondary antibodies for goat anti-rabbit immunoglobulin IgG horse radish peroxidase (HRP) and goat anti-mouse IgG HRP were obtained from Santa Cruz Biotechnology (CA, USA); TRIzol reagent was obtained from Invitrogen (CA, USA); PrimeScript ${ }^{\mathrm{TM}}$ RT Master Mix (Perfect Real Time) was purchased from TaKaRa (Dalian, China); Maxima SYBR Green qPCR Master were purchased from Thermo Scientific (CA, USA); ECL-chemiluminescent kit was obtained from Millipore (Billerica, MA,USA); Dimethyl sulfoxide (DMSO) and MTT (3-(4, 5-dimethylthiazol-2-yl)-2, 5-diphenyltetrazoliumbromide) were purchased from Sigma (Lee et al.).

\section{Cell culture and cell treatment with acetaldehyde}

The rat HSC line was obtained from KeyGEN BioTECH (Nanjing, China). HSCs were cultured in Dulbecco's modified Eagle's medium (DMEM, KeyGEN, China), and $10 \%$ fetal calf serum (FCS, Sijiqing, China). Cells were maintained at $37^{\circ} \mathrm{C}$ at an atmosphere of $5 \% \mathrm{CO}_{2}$. HSCs were cultured for $12 \mathrm{~h}$ and serum-starved with $0.5 \%$ FCS for $24 \mathrm{~h}$ before adding $200 \mu \mathrm{M}$ acetaldehyde.

\section{Cell proliferation assay}

HSCs $\left(5 \times 10^{3} / \mathrm{ml}\right)$ were seeded into each well of 96-well plates and then cultured in DMEM supplemented with other reagents for $48 \mathrm{~h}$. After culture, $5 \mathrm{mg} / \mathrm{ml} \mathrm{MTT}$ reagent was added to each well, and the plates were then cultured at $37{ }^{\circ} \mathrm{C}$ for $4 \mathrm{~h}$. 
Then DMSO was added to dissolve formazan crystals and examined in triplicate at $490 \mathrm{~nm}$ wavelength using a Thermomax microplate reader (bio-tek EL, USA).

\section{Cell cycle analysis}

To analyze the intracellular DNA content of HSCs which transfected EPAC siRNA, Cell Cycle Analysis Kit was used (Beyotime, China) according to the manufacturer's instruction. HSCs were fixed in $70 \%$ ethanol at $-20{ }^{\circ} \mathrm{C}$ overnight. Then cells were centrifuged at $3000 \mathrm{rpm}$ for 5 min and re-suspended in Phosphate Buffered Saline (PBS). After then, HSCs were stained with $0.5 \mathrm{ml}$ of propidium iodide (PI) staining buffer, including $200 \mathrm{mg} / \mathrm{ml} \mathrm{RNase} \mathrm{A,} 50 \mu \mathrm{g} / \mathrm{ml} \mathrm{PI}$, at $37{ }^{\circ} \mathrm{C}$ for $30 \mathrm{~min}$ in the dark. Analyses were performed on Cytomics FC500 MCL (Beckman coulter, USA).

\section{Western blot analysis}

Cells were washed with PBS thrice and lysed with RIPA (Beyotime, China) buffer containing protease inhibitor phenylmethylsulfonyl-fluoride (PMSF; Biosharp, USA). Protein concentrations was determined by the BCA protein assay kit (Boster, China). After separating by sodium dodecylsulfate polyacrylamide gel electrophoresis (10\%; $80 \mathrm{~V}$ for $30 \mathrm{~min}$ and then $120 \mathrm{~V}$ for $90 \mathrm{~min}$ ), the proteins were transferred onto a PVDF membrane (Millipore, USA). The blots were incubated overnight at $4{ }^{\circ} \mathrm{C}$ with primary antibodies diluted with TBS/Tween-20 (0.075\%) containing 3\% Marvel after blocking for $3 \mathrm{~h}$. The antibody of collagen type I, collagen type III, $\alpha$-SMA were diluted to 1:1000; EPAC1, EPAC2, Rap1GAP, Rap1 and $\beta$-actin were diluted to 1:500. Membranes were then washed with TBS/Tween-20 thrice, incubated with secondary antibodies (1:10000 diluted) for $1 \mathrm{~h}$ at room temperature. Finally repeating the washing again, protein bands was detected with enhanced chemiluminescence reagent (ECL; Thermo Scientific, USA). All experiments were performed at least three times with three independent protein extracts.

\section{Quantitative real-time PCR analysis (QRT-PCR)}

Total RNA was extracted from HSCs using TRIzol reagent. The first-strand cDNA was generated from total RNA using AMV Reverse Transcriptase

(Fermentas) according to the manufacturer's instructions. Quantitative real-time PCR analyses for mRNA of collagen type I, collagen type III, $\alpha$-SMA, EPAC1, EPAC2 
and $\beta$-actin were detected by PIKO REAL RT-PCR kits ( Thermo Scientific, USA). Primer sequences were designed as follows: collagen type I forward: GAT CCT GCC GAT GTC GCT AT and reverse: TGT AGG CTA GCT GTT CTT GCA; collagen type III forward: GGT TTG GAG AAT CTA TGA ATG GTG G and reverse: GCT GGA AAG AAG TCT GAG GAA GG; $\alpha$-SMA forward: CGA AGC GCA GAG CAA GAG A and reverse: CAT GTC GTC CCA GTT GGT GAT; EPAC1 forward: GTG AGC GAG ATA CCC GAC TTA G and reverse: GAT ACA TTC CCA CAG CCA TTC T; EPAC2 forward: CAT CCG AGT GCC TGT AGC CG and reverse: CAC CTT TTC TCC TCC AGA GTT CAT C; $\beta$-actin forward: CCC ATC TAT GAG GGT TAC GC and reverse: TTT AAT GTC ACG CAC GAT TTC.

\section{Rap1 Activation Assay}

The activation of Rap1 was examined by a Rap1 Activation Assay Kit (Neweastbio, USA). Briefly, The cell lysate was mixed with $20 \mu \mathrm{L}$ of resuspended protein A/G Agarose bead slurry with $1 \mu \mathrm{L}$ anti-active Rap1 monoclonal antibody and incubated at $4{ }^{\circ} \mathrm{C}$ for $1 \mathrm{~h}$ with gentle agitation. Following three washes in lysis buffer, the beads were suspended in $20 \mu \mathrm{L}$ of $2 \mathrm{X}$ reducing SDS-PAGE sample buffer. 15 $\mu \mathrm{L} /$ well of pull-down supernatant were loaded onto a $12 \%$ polyacrylamide gel and further analyzed with western blot using Rap1 specific antibody.

\section{RNA interference}

Small interfering RNA (siRNA) oligonucleotides against EPAC1, EPAC2 genes and negative control sequences were designed and synthesized by the Shanghai Gena Pharma (Shanghai, China) and contained the following sequences: EPAC1 siRNA forward: 5'-CCA GCA UUC UGAUCU ACA UTT -3' and reverse: 5'-AUG UAG AUCAGA AUG CUG GTT -3'; EPAC2 siRNA forward: 5'-GAG CGCCAU UUC CAA -3' and reverse: 5'-UUC AAC UUC AUA CAG CCA CTT-3'; negative control forward: 5'-UUC UCC GAA CGU GUC ACG UTT-3' and reverse: 5'-ACG UGA CAC GUU CGG AGA ATT-3'. Cells were transfected with siRNA using Lipofectamin2000 (Invitrogen, USA) according to the manufacturer's protocol. QRT-PCR and Western blot analysis were used to determine the efficiency of knockdown.

\section{Statistical Analysis}


All results are presented as the mean \pm SD. Differences between 2 groups were analyzed by 2-tailed Student's $t$ test, differences among $>2$ groups were analyzed by one-way ANOVA. $P<0.05$ was considered statistically significant.

\section{Results}

\section{Quantification of the expression of EPAC1 and EPAC2 in acetaldehyde-induced HSCs}

To investigate whether the expression of EPAC1 and EPAC2 were altered in acetaldehyde-induced HSCs, the QRT-PCR and western blotting were applied to quantify the levels of the mRNA and protein for EPAC1 and EPAC2. The HSCs were treated with $200 \mu \mathrm{M}$ acetaldehyde for $48 \mathrm{~h}$. Our results showed that the expression of EPAC2 at both protein and mRNA levels were significantly increased as compared with that of control group, whereas the expression of EPAC1 was decreased markedly by the addition of acetaldehyde (Fig. 1).

\section{Effect of EPAC on proliferation and activation of acetaldehyde-induced HSCs}

The HSCs were pretreated with acetaldehyde $(200 \mu \mathrm{M})$ for $24 \mathrm{~h}$, and co-cultured with Me-cAMP $(50 \mu \mathrm{M})$ for $24 \mathrm{~h}$. As shown in Fig. 2a, Me-cAMP restored the expression of EPAC1, and the expression EPAC2 was slightly increased. MTT assay showed that Me-cAMP displayed remarkable inhibition of the proliferation in acetaldehyde-induced HSCs (Fig. 3a). As shown in Fig. 3b, the results of cell cycle analysis showed that Me -cAMP increased the ratio of cells in the G0/G1 phase and decreased those in the $\mathrm{S}$ and G2/M phase. There were significant differences in the ratio of cells in G0/G1 phase and S plus G2/M phase between Me-cAMP-treated groups and model group. Furthermore, addition of Me-cAMP potently decreased the mRNA and protein expression of $\alpha$-SMA, collagen type I and III in acetaldehyde-induced HSCs (Fig. 2b).

To gain insight into the potential signaling pathways mediated by EPAC, the protein levels of both Rap1-GTP and total Rap1 were determined. As shown in Fig. $3 \mathrm{c}$, the expression of Rap1-GTP were significantly downregulated by acetaldehyde but upregulated by Me-cAMP, whereas total Rap1 protein levels remained unchanged, indicating that EPAC activation by Me-cAMP enhanced the expression of GTP-bound (activated) Rap1. 


\section{Effect of EPAC siRNA on proliferation and activation of acetaldehyde-induced} HSCs

To further dissect the functional role of EPAC1 and EPAC2, the small interfering RNA was used. After $48 \mathrm{~h}$ following the transfection, the cells were photographed at $\times 920$ magnification by fluorescence microscope (data not shown). Transfection efficiency detected by western blot (Fig. 4) and QRT-PCR (data of QRT-PCR was not shown). As shown in Fig. 5a, EPAC2 siRNA significantly inhibited the proliferation of HSCs, whereas EPAC1 siRNA and double knockdown of EPAC1 and 2 remarkably increased the rate of HSC proliferation. In accordance with the findings from previous studies, the results of cell cycle analysis showed that only EPAC2 siRNA enhanced the ratio of cells in the G0/G1 phase and decreased those in the S and G2/M phase apparently (Fig. 5b). In addition, double knockdown of EPAC1 and 2 had a profound promotion of the expression of $\alpha$-SMA and collagen type I and type III (Fig. 6).

QRT-PCR and western blot results indicated that knockdown of EPAC1 further enhanced acetaldehyde-induced $\alpha$-SMA and collagen type I and III mRNA and protein levels (Fig. 7a). However, EPAC2 depletion prevented the increase of both collagen type I and III, and inhibited acetaldehyde-induced $\alpha$-SMA expression strongly (Fig. 7b). Taken together, these data suggested that the activation and proliferation of HSCs could be suppressed by EPAC1 and promoted by EPAC2.

\section{EPAC and PKA activation had opposite effects on synthesis of collagen and a-SMA in acetaldehyde-induced HSCs}

To better understand the roles of individual cAMP effectors EPAC and PKA in the regulation of HSCs activation induced by acetaldehyde, we applied the selective PKA activator Phe-cAMP and the selective EPAC activator Me-cAMP. Consistent with our previous findings, treatment of acetaldehyde-induced HSCs with Me-cAMP significantly decreased the protein expression of markers of HSC activation, including $\alpha$-SMA, collagen type I and III. In contrast, the activation of PKA obviously enhanced the expression of the HSCs activation marker (Fig. 8a). In addition, we also assessed the role of Rap1, a downstream target of EPAC, on EPAC-regulated activation of acetaldehyde-induced HSCs . EPAC activation by Me-cAMP increased the expression of GTP-bound (activated) Rap1, while PKA 
activation by Phe-cAMP had negligible effects on such binding (Fig. 8b). These data further demonstrated that EPAC could play a protective role against acetaldehyde-induced HSCs activation via Rap1.

\section{Discussion}

ALD is a major health problem worldwide and ALF is a turning point in the development of ALD. HSCs are the primary source of excess ECM proteins in liver fibrosis (Kocabayoglu and Friedman 2013). In normal liver, HSCs is localized in the space of disse and store vitamin A in the form of retinyl esters (Friedman et al. 1985). During the development of fibrosis, HSCs undergo a process of activation acquiring a myofibroblast-like phenotype characterized by $\alpha$-SMA expression and increased proliferation and synthesis of ECM components, including collagen type I and III (Svegliati-Baroni et al. 2001). Ethanol has been reported to induce liver fibrosis via direct fibrotic effects of acetaldehyde on HSCs (Svegliati-Baroni et al. 2001). Draw on the previous research, cell culture model of alcoholic liver fibrosis was successfully constructed by incubating the HSCs with $200 \mu \mathrm{M}$ acetaldehyde for $48 \mathrm{~h}$, which may closely mimic the activation of HSCs in vivo (Wang et al. 2014; Yang et al. 2015).

Experiments have shown that the cyclooxygenase-2 (COX-2)/PGE2 signaling mechanism was involved in mediating proliferation of $\mathrm{HSC}$, and COX-2 specific inhibitors could attenuate hepatic fibrosis probably via COX-2/PGE2/cAMP pathway (Hui et al. 2004; Zhao et al. 2012). Previous studies have also revealed the remarkably enhanced expression of COX-2 in experimental ALD (Nanji et al. 1997). In our prior studies, we found that activated HSCs isolated from alcohol induced fibrotic rat liver displayed a dramatic upregulation of the cAMP/PKA/CREB signaling pathway, which was positively correlated with the progression of liver fibrosis (Wang et al. 2015), indicating that the COX-2/PGE2/cAMP pathways may have major effects on the activation of HSCs induced by acetaldehyde.

The effects of cAMP are mediated by two ubiquitously expressed cAMP receptors: the classical PKA and the recently discovered EPAC. EPAC1 and EPAC2 are multidomain proteins, the biggest structural difference between EPAC1 and EPAC2 is the existence of an additional cyclic nucleotide-binding domain (CNBD) within the $\mathrm{N}$ terminus of EPAC2 (Banerjee and Cheng 2015; Kawasaki et al. 1998). 
The extra part exhibits a reduced affinity for cAMP and is incapable to induce GEF activity following cAMP binding. EPAC1 and EPAC2 share high structural homology throughout their regulatory and catalytic domains despite this difference (Parnell et al. 2015; Rehmann et al. 2008). Upon cAMP binding, EPAC undergoes a conformational change that accepts the interaction of its dominating catalytic domain with Rap, resulting in Rap activation and subsequent downstream effects (Gloerich and Bos 2010). So far, EPAC proteins are implicated in several diverse tissue responses. Yokoyama et al. reported that TGF $\beta 1$ decreases transcriptional expression of EPAC1 while the overexpression of EPAC1 inhibits TGF $\beta 1$-induced collagen synthesis in fibroblasts (Yokoyama et al. 2008b), and recent study shows that deletion of EPAC1 in T cells leads to an increased level of SMAD7, a reduced expression of SMAD4 and a suppressed SMAD2 phosphorylation in response to TGF $\beta 1$ treatment (Almahariq et al. 2015).

In our article, QRT-PCR and western blot were used to quantify both protein and mRNA levels of the two isoforms of EPAC. Our results showed that both two isoforms could be present in rat HSCs. Furthermore, the expression of EPAC2 was significantly increased in acetaldehyde-induced HSCs as compared with that of control group, whereas the expression of EPAC1 was down-regulated by the addition of acetaldehyde. These results suggested that perhaps EPAC1 or EPAC2 may in part responsible for regulating HSCs activation, such as the production of $\alpha$-SMA and collagen type I and III.

According to the previous results, the analog of natural cAMP, Me-cAMP, was used. It is more than tenfold as efficient as cAMP in activating EPAC1 in vitro, and results in a threefold-higher maximum activity of EPAC1, causing this analog a so-called superagonist for EPAC1 (Rehmann et al. 2003). Whereas EPAC activation not only apparently reduced the expression of $\alpha$-SMA, collagen type I and III production as compared with the model group, but also suppressed the proliferation of HSCs. Hence we speculate that the reduction caused by activation of EPAC, perhaps by EPAC1 to a large extent, though acetaldehyde induced enhancive expression of EPAC2. In other words, blunt the decrease in EPAC expression, especially EPAC1, may thus be strategies to block or reverse alcoholic liver fibrosis.

To gain insight into the pathways mediated by EPAC, the protein levels of both Rap1-GDP and total Rap1 were determined. EPAC have been viewed as a family of 
cAMP-responsive GEFs for Rap1 (Li et al. 2015). Data from our recent study showed that Me-cAMP upregulated it while total Rap1 proteins remained unchanged.

To elucidate the mechanism of the impact of EPAC, we silenced EPAC1 or EPAC2 and found that EPAC1 silencing, but not EPAC2, increased acetaldehyde-induced expression of $\alpha$-SMA, collagen type I and III and the proliferation of HSCs, which is consistent with the observation of Miguel et al. (Perez-Aso et al. 2013). Paradoxically, knockdown of both EPAC1 and EPAC2 to the acetaldehyde-induced HSCs showed the same effect as knockdown of EPAC1. Up to now, it is reported that EPAC1 overexpression can lead to cardiac hypertrophy whereas EPAC2 activation can be arrhythmogenic, in part because of distinct subcellular localization, and EPAC1 is localized and functionally involved in nuclear signaling, however EPAC2 is located at the T tubules and regulates arrhythmogenic sarcoplasmic reticulum Ca leak (Pereira et al. 2015). But why EPAC1 and EPAC2 contribute to differential functional effects in the activation of HSCs is not clealy defined.

In 2014, the scientists observed that Leishmania facilitates an immunosuppressive environment in macrophages by COX2/PGE2/EP2/cAMP signaling that is differentially regulated by PKA and EPAC (Saha et al. 2014). Results from these studies provided a novel insight into mechanisms by which EPAC and PKA may have independent, or even opposing effects on acetaldehyde-induced HSCs. The physiologically relevant cAMP affinities for EPAC vs. PKA have not been clearly defined. However, the available data are conflictive on this issue, some researchers declare that stimulation of the cAMP/PKA signaling pathway inhibits proliferation of activated HSCs (Acquaviva et al. 2013; Houglum et al. 1997), and recent study demonstrated that both EPAC and PKA activation inhibit collagen type I and III expression and DNA synthesis (Yokoyama et al. 2008b). But we and others have found that the expression $\alpha$-SMA, collagen type I and III were inhibited by EPAC activator, Me-cAMP and promoted by PKA activator, Phe-cAMP. Perhaps due to paradoxical effect of cAMP. There is evidence that that physiological levels of cAMP resulting from pharmacologic A2AR stimulation promote fibroblast proliferation and collagen production via PKA-dependent pathway, but that the high levels of cAMP, beyond the physiological range that can be achieved by activation of adenylate cyclase $(\mathrm{AC})$ by forskolin, inhibit fibroblast proliferation and collagen production via EPAC/Rap1 pathway (Perez-Aso et al. 2014). 
In conclusion, in this study we used acetaldehyde-induced HSCs culture as the alcoholic liver fibrosis model to investigate the role of EPAC. The current data showed that both EPAC1 and EPAC2 were present in rat HSCs, and EPAC1 probably exerted more protective influence via Rap1; increasing EPAC activity by Me-cAMP could inhibit profibrotic response and EPAC2 depletion, but not EPAC1, prevented proliferation of HSCs and acetaldehyde-induced increase in $\alpha$-SMA and collagen type I and III. Moreover, PKA and EPAC activation perhaps had opposite effects on synthesis of collagen in acetaldehyde-induced HSCs and PKA activation had negligible effects on the binding between Rap1 and GTP. This study provided a new insight into the role of EPAC in the pathogenesis of alcohol-induced liver fibrosis and will help to develop a rational use of EPAC agonist-based therapy.

\section{Conflict of interest}

The authors declare that there are no conflicts of interest.

\section{Acknowledgments}

This project was supported by the National Science Foundation of China (No. 812704 98), Anhui Provincial Natural Science Foundation (No. 1104060 6M194) and Anhui Provincial Key Projects of Scientific Research in Universities (No. KJ2012A14 8).

\section{Refercences}

Acquaviva, A., Vecchio, D., Arezzini, B., Comporti, M., and Gardi, C. 2013.

Signaling pathways involved in isoprostane-mediated fibrogenic effects in rat hepatic stellate cells. Free Radic. Biol. Med. 65: 201-207. doi:

10.1016/j.freeradbiomed.2013.06.023. PMID: 23792773.

Almahariq, M., Mei, F.C., Wang, H., Cao, A.T., Yao, S., Soong, L., et al. 2015.

Exchange protein directly activated by cAMP modulates regulatory T-cell-mediated immunosuppression. Biochem. J. 465(2): 295-303. doi: 10.1042/BJ20140952. PMID: 25339598.

Banerjee, U., and Cheng, X. 2015. Exchange protein directly activated by cAMP encoded by the mammalian rapgef3 gene: Structure, function and therapeutics. Gene, doi: 10.1016/j.gene.2015.06.063. PMID: 26119090.

Boniface, K., Bak-Jensen, K.S., Li, Y., Blumenschein, W.M., McGeachy, M.J., McClanahan, T.K., et al. 2009. Prostaglandin E2 regulates Th17 cell differentiation 
and function through cyclic AMP and EP2/EP4 receptor signaling. J. Exp. Med. 206(3): 535-548. doi: 10.1084/jem.20082293. PMID: 19273625.

Breckler, M., Berthouze, M., Laurent, A.C., Crozatier, B., Morel, E., and Lezoualc'h, F. 2011. Rap-linked cAMP signaling Epac proteins: compartmentation, functioning and disease implications. Cellular signalling, 23(8): 1257-1266. doi:

10.1016/j.cellsig.2011.03.007. PMID: 21402149.

Collart, F., de Timary, P., Dom, G., Dor, B.D., Duprez, D., Lengele, J.P., et al. 2015. Alcohol-induced hypertension: an important healthcare target in Belgium. Acta clinica Belgica, 2295333715Y0000000039. doi: 10.1179/2295333715Y.0000000039. PMID: 26135944.

de Rooij, J., Zwartkruis, F.J., Verheijen, M.H., Cool, R.H., Nijman, S.M., Wittinghofer, A., et al. 1998. Epac is a Rap1 guanine-nucleotide-exchange factor directly activated by cyclic AMP. Nature, 396(6710): 474-477. doi: 10.1038/24884. PMID: 9853756.

Duddempudi, A.T. 2012. Immunology in alcoholic liver disease. Clin Liver Dis 16(4): 687-698. doi: 10.1016/j.cld.2012.08.003. PMID: 23101977.

Friedman, S.L. 1999. Stellate cell activation in alcoholic fibrosis--an overview. Alcohol.: Clin. Exp. Res. 23(5): 904-910. PMID: 10371412.

Friedman, S.L., Roll, F.J., Boyles, J., and Bissell, D.M. 1985. Hepatic lipocytes: the principal collagen-producing cells of normal rat liver. Proc. Natl. Acad. Sci. U.S.A 82(24): 8681-8685. PMID: 3909149.

Gao, B., and Bataller, R. 2011. Alcoholic liver disease: pathogenesis and new therapeutic targets. Gastroenterology, 141(5): 1572-1585. doi:

10.1053/j.gastro.2011.09.002. PMID: 21920463.

Gao, B., Seki, E., Brenner, D.A., Friedman, S., Cohen, J.I., Nagy, L., et al. 2011. Innate immunity in alcoholic liver disease. Am. J. Physiol. 300(4): G516-G525. doi: 10.1152/ajpgi.00537.2010. PMID: 23075869.

Gloerich, M., and Bos, J.L. 2010. Epac: defining a new mechanism for cAMP action. Annu. Rev. Pharmacol. Toxicol. 50: 355-375. doi:

10.1146/annurev.pharmtox.010909.105714. PMID: 20055708.

Gualde, N., and Harizi, H. 2004. Prostanoids and their receptors that modulate dendritic cell-mediated immunity. Immunol Cell Biol 82(4): 353-360. doi:

10.1111/j.0818-9641.2004.01251.x. PMID: 15283844. 
Hernandez-Gea, V., and Friedman, S.L. 2011. Pathogenesis of liver fibrosis. Annual review of pathology 6: 425-456. doi: 10.1146/annurev-pathol-011110-130246. PMID: 21073339.

Houglum, K., Lee, K.S., and Chojkier, M. 1997. Proliferation of hepatic stellate cells is inhibited by phosphorylation of CREB on serine 133. J. Clin. Invest. 99(6): 1322-1328. doi: 10.1172/JCI119291. PMID: 9077542.

Hui, A.Y., Cheng, A.S.-L., Chan, H.L.-Y., Go, M.Y.-Y., Chan, F.K.-L., Sakata, R., et al. 2004. Effect of prostaglandin E2 and prostaglandin I2 on PDGF-induced proliferation of LI90, a human hepatic stellate cell line. Prostaglandins, Leukotrienes and Essential Fatty Acids (PLEFA), 71(5): 329-333. doi: 10.1016/j.plefa.2004.04.007. PMID: 15380820.

Insel, P.A., Murray, F., Yokoyama, U., Romano, S., Yun, H., Brown, L., et al. 2012. cAMP and Epac in the regulation of tissue fibrosis. Br. J. Pharmacol. 166(2): 447-456. doi: 10.1111/j.1476-5381.2012.01847.x. PMID: 22233238.

Jeyaraj, S.C., Unger, N.T., and Chotani, M.A. 2011. Rap1 GTPases: an emerging role in the cardiovasculature. Life SCI. 88(15-16): 645-652. doi: 10.1016/j.lfs.2011.01.023. PMID: 21295042.

Kawasaki, H., Springett, G.M., Mochizuki, N., Toki, S., Nakaya, M., Matsuda, M., et al. 1998. A family of cAMP-binding proteins that directly activate Rap1. Science, 282(5397): 2275-2279. PMID: 9856955.

Kocabayoglu, P., and Friedman, S.L. 2013. Cellular basis of hepatic fibrosis and its role in inflammation and cancer. Front Biosci (Schol Ed) 5: 217-230. PMID: 23277047.

Lachenmeier, D.W., Monakhova, Y.B., and Rehm, J. 2014. Influence of unrecorded alcohol consumption on liver cirrhosis mortality. World J. Gastroenterol. J 20(23): 7217-7222. doi: 10.3748/wjg.v20.i23.7217. PMID: 24966592.

Lee, L.N., Susarla, S.M., M, H.H., Henstrom, D.K., Cheney, M.L., and Hadlock, T.A. 2013. A comparison of facial nerve grading systems. Ann Plast Surg 70(3): 313-316. doi: 10.1097/SAP.0b013e31826acb2c. PMID: 23241802.

Li, Z., Liu, X.B., Liu, Y.H., Xue, Y.X., Wang, P., Liu, L.B., Yao, Y.L., and Ma, J. 2015. Functions for the cAMP/Epac/Rap1 Signaling Pathway in Low-Dose Endothelial Monocyte-Activating Polypeptide-II-Induced Opening of Blood-Tumor Barrier. J. Mol. Neurosci. doi: 10.1007/s12031-015-0594-6. PMID: 26044663. 
Luo, Z., Liu, H., Sun, X., Guo, R., Cui, R., Ma, X., et al. 2013. RNA interference against discoidin domain receptor 2 ameliorates alcoholic liver disease in rats. PloS One 8(2): e55860. doi: 10.1371/journal.pone.0055860. PMID: 23409069.

Mello, T., Ceni, E., Surrenti, C., and Galli, A. 2008. Alcohol induced hepatic fibrosis: role of acetaldehyde. Mol Aspects Med 29(1-2): 17-21. doi:

10.1016/j.mam.2007.10.001. PMID: 18164754.

Nanji, A.A., Miao, L., Thomas, P., Rahemtulla, A., Khwaja, S., Zhao, S., et al. 1997. Enhanced cyclooxygenase-2 gene expression in alcoholic liver disease in the rat. Gastroenterology, 112(3): 943-951. PMID: 9041257.

Okumura, S., Fujita, T., Cai, W., Jin, M., Namekata, I., Mototani, Y., et al. 2014. Epac1-dependent phospholamban phosphorylation mediates the cardiac response to stresses. J. Clin. Invest. 124(6): 2785-2801. doi: 10.1172/JCI64784. PMID: 24892712.

Orman, E.S., Odena, G., and Bataller, R. 2013. Alcoholic liver disease: pathogenesis, management, and novel targets for therapy. J. Gastroenterol. Hepatol. 28 Suppl 1: 77-84. doi: 10.1111/jgh.12030. PMID: 23855300.

Parnell, E., Palmer, T.M., and Yarwood, S.J. 2015. The future of EPAC-targeted therapies: agonism versus antagonism. Trends Pharmacol. Sci. 36(4): 203-214. doi: 10.1016/j.tips.2015.02.003. PMID: 25744542.

Pereira, L., Rehmann, H., Lao, D.H., Erickson, J.R., Bossuyt, J., Chen, J., et al. 2015. Novel Epac fluorescent ligand reveals distinct Epac1 vs. Epac2 distribution and function in cardiomyocytes. Proc. Natl. Acad. Sci. U.S.A. 112(13): 3991-3996. doi: 10.1073/pnas.1416163112. PMID: 25829540.

Perez-Aso, M., Fernandez, P., Mediero, A., Chan, E.S., and Cronstein, B.N. 2014. Adenosine $2 \mathrm{~A}$ receptor promotes collagen production by human fibroblasts via pathways involving cyclic AMP and AKT but independent of Smad2/3. FASEB J. 28(2): 802-812. doi: 10.1096/fj.13-241646. PMID: 24200882.

Perez-Aso, M., Mediero, A., and Cronstein, B.N. 2013. Adenosine A2A receptor (A2AR) is a fine-tune regulator of the collagen1:collagen3 balance. Purinergic signalling, 9(4): 573-583. doi: 10.1007/s11302-013-9368-1. PMID: 23749290. Ramaiah, S., Rivera, C., and Arteel, G. 2004. Early-phase alcoholic liver disease: an update on animal models, pathology, and pathogenesis. Int J Toxicol 23(4): 217-231. doi: 10.1080/10915810490502069. PMID: 15371166. 
Rehmann, H., Arias-Palomo, E., Hadders, M.A., Schwede, F., Llorca, O., and Bos, J.L. 2008. Structure of Epac2 in complex with a cyclic AMP analogue and RAP1B. Nature, 455(7209): 124-127. doi: 10.1038/nature07187. PMID: 18660803.

Rehmann, H., Schwede, F., Doskeland, S.O., Wittinghofer, A., and Bos, J.L. 2003. Ligand-mediated activation of the cAMP-responsive guanine nucleotide exchange factor Epac. J. Biol. Chem. 278(40): 38548-38556. doi: 10.1074/jbc.M306292200. PMID: 12888551.

Roberts, O.L., and Dart, C. 2014. cAMP signalling in the vasculature: the role of Epac (exchange protein directly activated by cAMP). Biochem. Soc. Trans. 42(1): 89-97. doi: 10.1042/BST20130253. PMID: 24450633.

Roscioni, S.S., Elzinga, C.R., and Schmidt, M. 2008. Epac: effectors and biological functions. Naunyn-Schmiedeberg's Arch. Pharmacol. 377(4-6): 345-357. doi:

10.1007/s00210-007-0246-7. PMID: 18176800.

Saha, A., Biswas, A., Srivastav, S., Mukherjee, M., Das, P.K., and Ukil, A. 2014.

Prostaglandin E2 Negatively Regulates the Production of Inflammatory

Cytokines/Chemokines and IL-17 in Visceral Leishmaniasis. J. Immunol. 193(5): 2330-2339. doi: 10.4049/jimmunol.1400399. PMID: 25049356.

Schmidt, M., Dekker, F.J., and Maarsingh, H. 2013. Exchange protein directly activated by cAMP (epac): a multidomain cAMP mediator in the regulation of diverse biological functions. Pharmacol. Rev. 65(2): 670-709. doi: 10.1124/pr.110.003707. PMID: 23447132.

Serezani, C.H., Ballinger, M.N., Aronoff, D.M., and Peters-Golden, M. 2008. Cyclic AMP: master regulator of innate immune cell function. Am J Respir Cell Mol Biol 39(2): 127-132. doi: 10.1165/rcmb.2008-0091TR. PMID: 18323530.

Svegliati-Baroni, G., Ridolfi, F., Di Sario, A., Saccomanno, S., Bendia, E., Benedetti, A., et al. 2001. Intracellular signaling pathways involved in acetaldehyde-induced collagen and fibronectin gene expression in human hepatic stellate cells. Hepatology, 33(5): 1130-1140. doi: 10.1053/jhep.2001.23788. PMID: 11343241.

Tarantino, G. 2011. Spleen: A new role for an old player? World J Gastroentero 17(33): 3776. doi: 10.3748/wjg.v17.i33.3776. PMID: 21987619.

Tonk, E.C., de Groot, D.M., Wolterbeek, A.P., Penninks, A.H., Waalkens-Berendsen, I.D., Piersma, A.H., and van Loveren, H. 2013. Developmental immunotoxicity of ethanol in an extended one-generation reproductive toxicity study. Arch Toxicol 87(2): 323-335. doi: 10.1007/s00204-012-0940-1. PMID: 23007559. 
Ueno, H., Shibasaki, T., Iwanaga, T., Takahashi, K., Yokoyama, Y., Liu, L.M., Yokoi, N., Ozaki, N., Matsukura, S., Yano, H., and Seino, S. 2001. Characterization of the gene EPAC2: structure, chromosomal localization, tissue expression, and identification of the liver-specific isoform. Genomics, 78(1-2): 91-98. doi: 10.1006/geno.2001.6641. PMID: 11707077.

Wang, H., Guan, W., Yang, W., Wang, Q., Zhao, H., Yang, F., et al. 2014. Caffeine inhibits the activation of hepatic stellate cells induced by acetaldehyde via adenosine A2A receptor mediated by the cAMP/PKA/SRC/ERK1/2/P38 MAPK signal pathway. PloS One 9(3): e92482. doi: 10.1371/journal.pone.0092482. PMID: 24682220.

Wang, Q., Dai, X., Yang, W., Wang, H., Zhao, H., Yang, F., et al. 2015. Caffeine protects against alcohol-induced liver fibrosis by dampening the cAMP/PKA/CREB pathway in rat hepatic stellate cells. Int. Immunopharmacol. 25(2): 340-352. doi: 10.1016/j.intimp.2015.02.012. PMID: 25701503.

Yang, Y., Wang, H., Lv, X., Wang, Q., Zhao, H., Yang, F., et al. 2015. Involvement of cAMP-PKA pathway in adenosine A1 and A2A receptor-mediated regulation of acetaldehyde-induced activation of HSCs. Biochimie, doi:

10.1016/j.biochi.2015.04.019. PMID: 25956975.

Yokoyama, U., Minamisawa, S., Quan, H., Akaike, T., Suzuki, S., Jin, M., et al. 2008a. Prostaglandin E2-activated Epac promotes neointimal formation of the rat ductus arteriosus by a process distinct from that of cAMP-dependent protein kinase A. J. Biol. Chem. 283(42): 28702-28709. doi: 10.1074/jbc.M804223200. PMID: 18697745.

Yokoyama, U., Patel, H.H., Lai, N.C., Aroonsakool, N., Roth, D.M., and Insel, P.A. 2008b. The cyclic AMP effector Epac integrates pro- and anti-fibrotic signals. Proc. Natl. Acad. Sci. U.S.A. 105(17): 6386-6391. doi: 10.1073/pnas.0801490105. PMID: 18434542.

Zhao, Y., Wang, Y., Wang, Q., Liu, Z., Liu, Q., and Deng, X. 2012. Hepatic stellate cells produce vascular endothelial growth factor via phospho-p44/42 mitogen-activated protein kinase/cyclooxygenase-2 pathway. Mol. Cell. Biochem 359(1-2): 217-223. doi: 10.1007/s11010-011-1016-x. PMID: 21863308. 


\section{Figure captions}

Fig. 1 The expression of EPAC1 and EPAC2 in control group and model group ${ }^{\#} P<0.05,{ }^{\# \#} P<0.01$ vs. control group.

Fig. 2 a Me-cAMP resulted in up-regulation of EPAC1 and EPAC2 in both mRNA and protein levels.

b Western blot analysis and QRT-PCR indicated that the application of Me-cAMP resulted in down-regulation of collagen type I and III, $\alpha$-SMA .

${ }^{\#} P<0.05,{ }^{\#} P<0.01$ vs. control group; ${ }^{*} P<0.05,{ }^{* *} P<0.01,{ }^{* * *} P<0$.

001 vs. model group.

Fig. 3 a Cell proliferation was determined by MTT assay.

b Cell cycle analysis of HSCs treated with acetaldehyde and Me-cAMP.

c Rap1 activation (GTP-bound) was probed by western blot.

${ }^{\#} P<0.05,{ }^{\# \#} P<0.001$ vs. control group; ${ }^{*} P<0.05,{ }^{* *} P<0.01,{ }^{* * *} P<0$.

001 vs. model group.

Fig. 4 siRNAs for EPAC1, EPAC2, and double knockdown EPAC1+2 selectively reduce the protein expression when compared to scrambled siRNA group. ${ }^{\# \#} P<0.01$ vs. control group; ${ }^{* *} P<0.01$ vs. scrambled siRNA group.

Fig. 5 a Cell proliferation was detected by MTT assay.

b The role of EPAC siRNA on cell cycle in acetaldehyde-induced HSCs.

${ }^{\# \#} P<0.01$ vs. control group; ${ }^{*} P<0.05,{ }^{* *} P<0.01,{ }^{* * *} P<0.001$ vs. scrambled siRNA group. 
Fig. 6 Impact of double knockdown of EPAC1+2 on the expression of $\alpha$-SMA, collagen type I and III in acetaldehyde-induced HSCs.

${ }^{\# \#} P<0.01$ vs. control group; ${ }^{*} P<0.05,{ }^{* *} P<0.01$ vs. scrambled siRNA group.

Fig. 7 Impact of EPAC1 or 2 silencing on the expression of $\alpha$-SMA, collagen type I and III in acetaldehyde-induced HSCs.

${ }^{\#} P<0.05,{ }^{\# \#} P<0.01$ vs. control group; ${ }^{*} P<0.05,{ }^{* *} P<0.01$ vs. scrambled siRNA group.

Fig. 8 a Impact of EPAC and PKA activation on acetaldehyde-increased $\alpha$-SMA, collagen I and III expression.

b Rap1-GDP and total Rap1 was detected in addition of Me-cAMP and Phe-cAMP ${ }^{\#} P<0.05,{ }^{\# \#} P<0.01$ vs. control group; ${ }^{*} P<0.05,{ }^{* *} P<0.01$ vs. model group. 

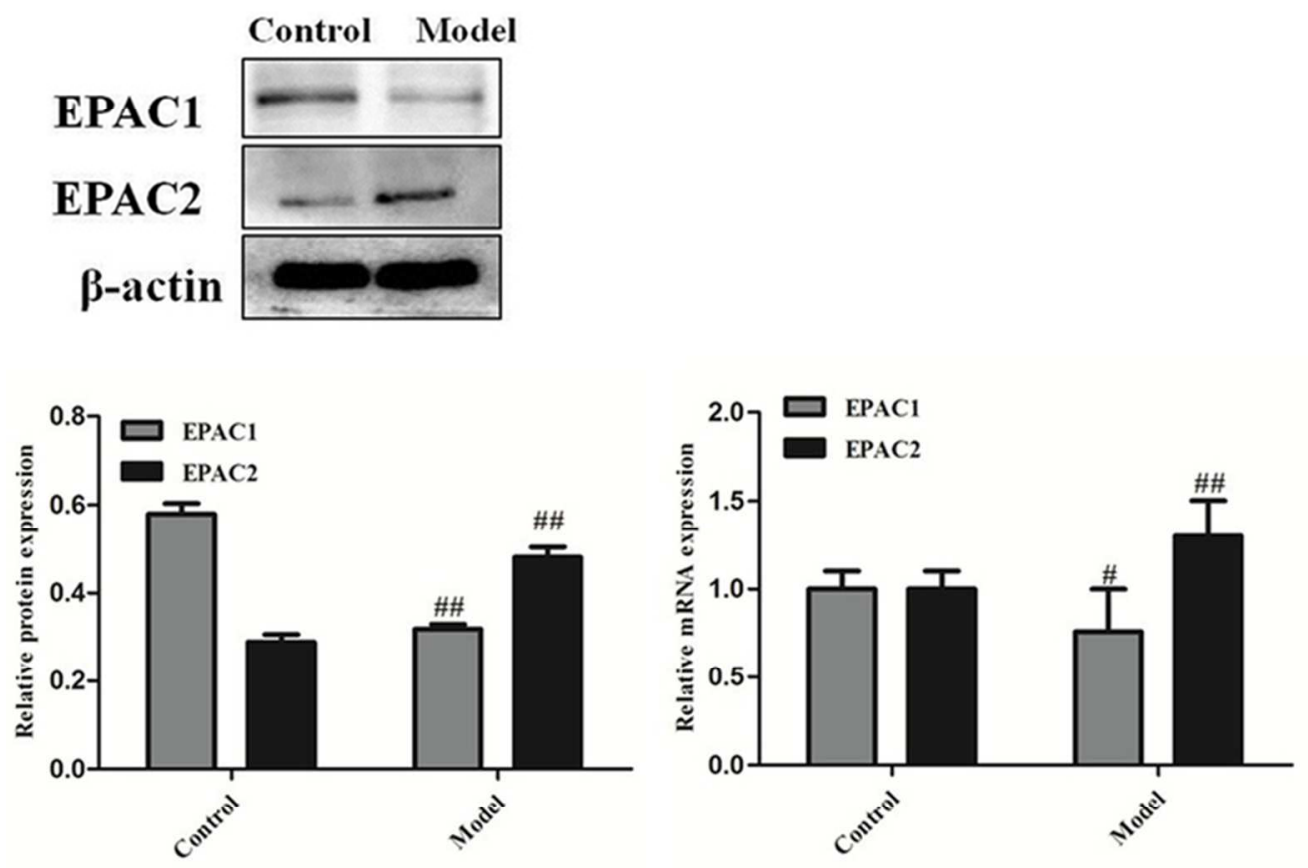

$63 \times 47 \mathrm{~mm}(300 \times 300 \mathrm{DPI})$ 

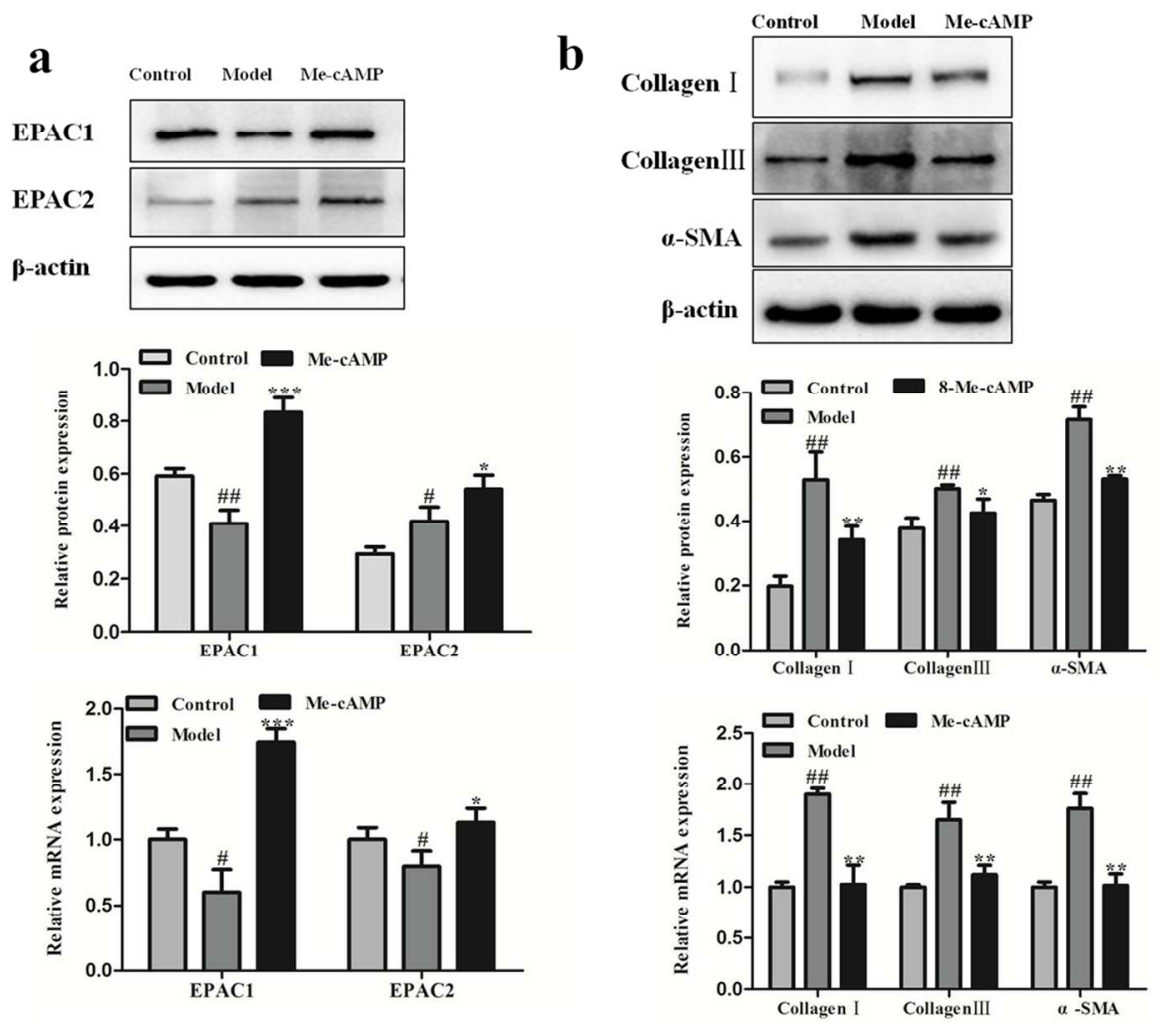

$129 \times 103 \mathrm{~mm}(300 \times 300$ DPI $)$ 

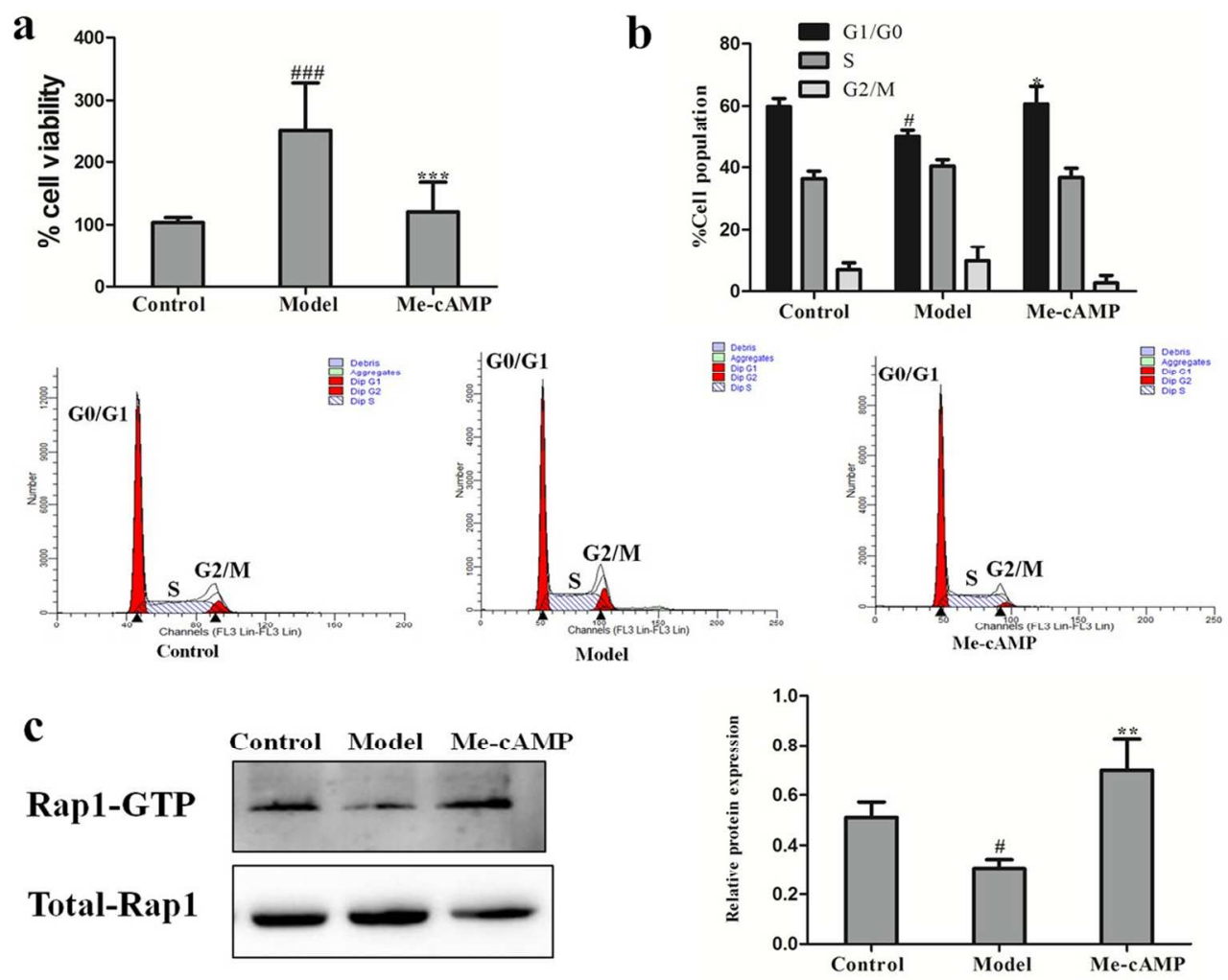

$129 \times 103 \mathrm{~mm}(300 \times 300 \mathrm{DPI})$ 

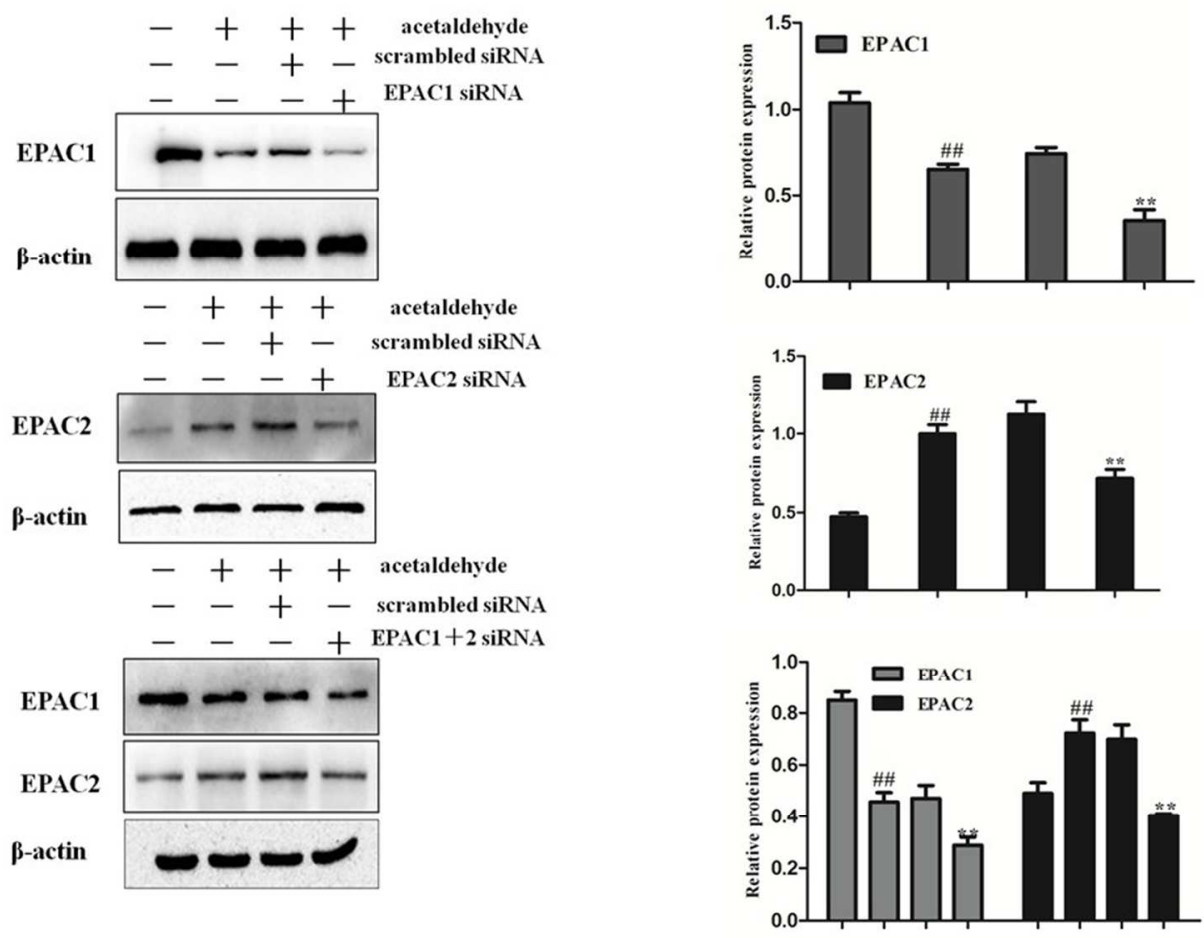

$83 \times 63 \mathrm{~mm}(300 \times 300 \mathrm{DPI})$ 


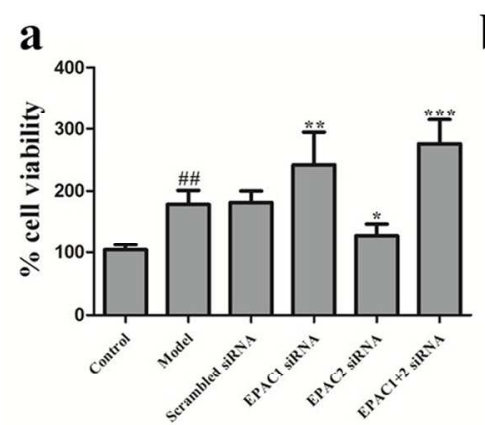

b
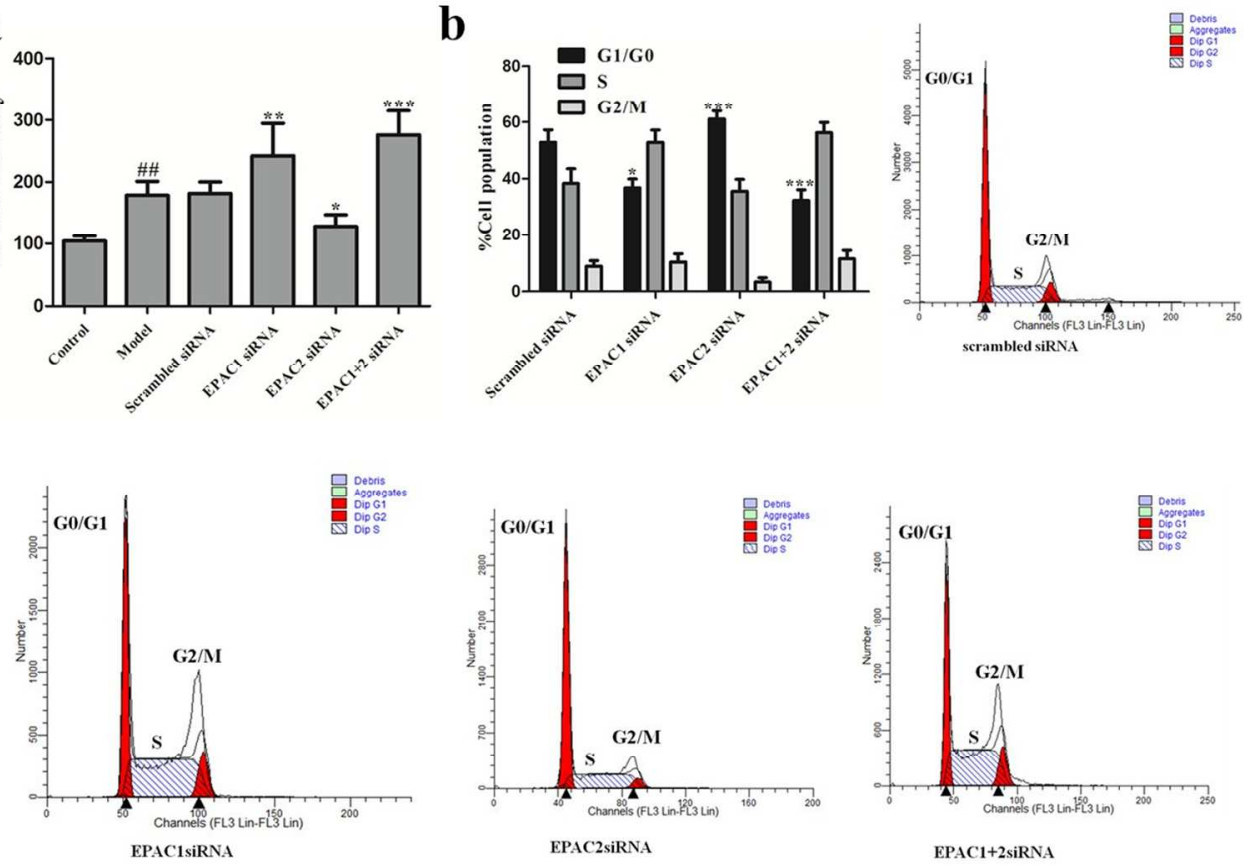

$129 \times 103 \mathrm{~mm}(300 \times 300 \mathrm{DPI})$ 

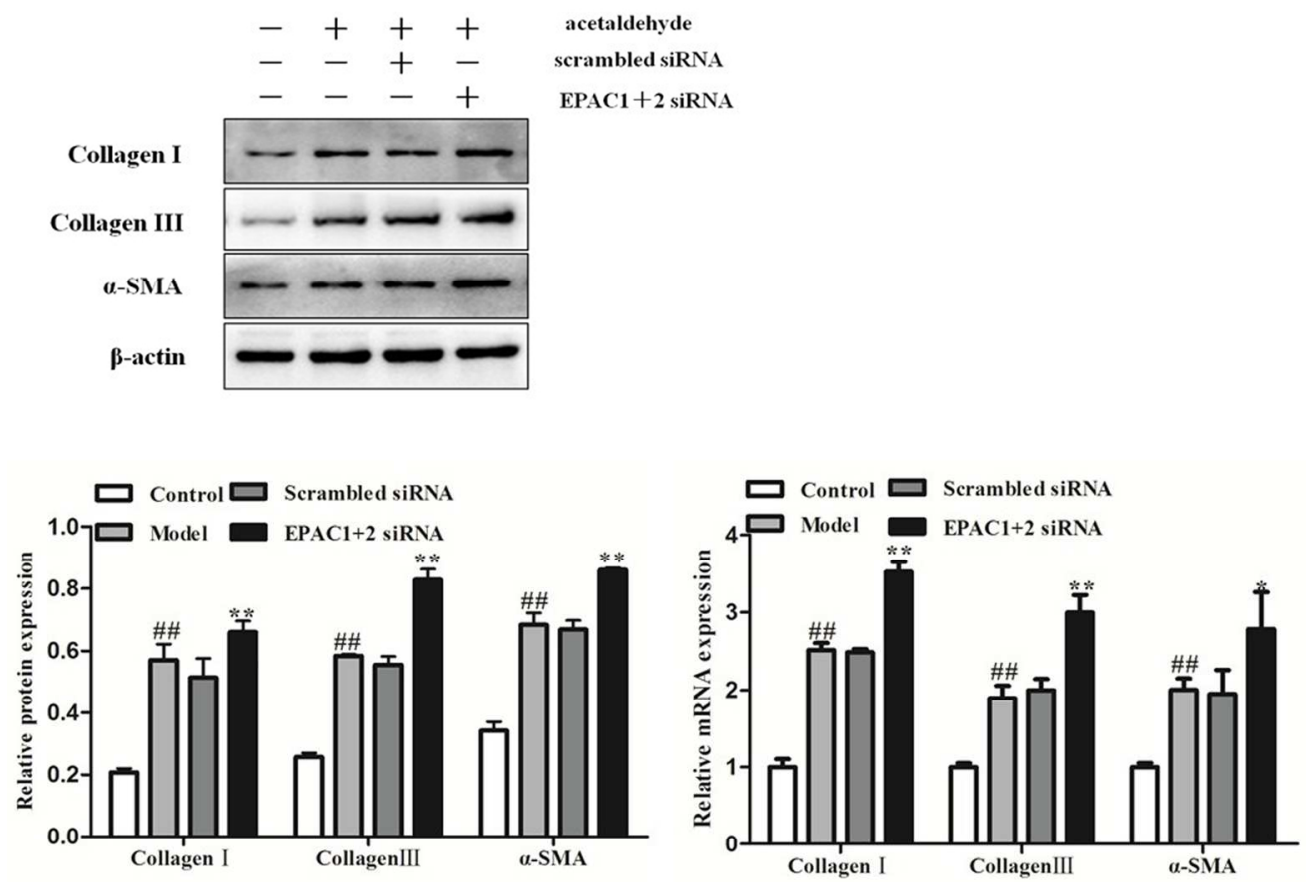

$129 \times 96 \mathrm{~mm}(300 \times 300$ DPI $)$ 


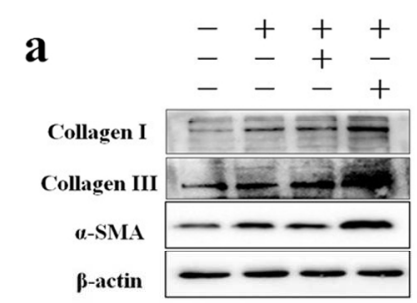

acetaldehyde scrambled siRNA b

b EPAC1 SiRNA
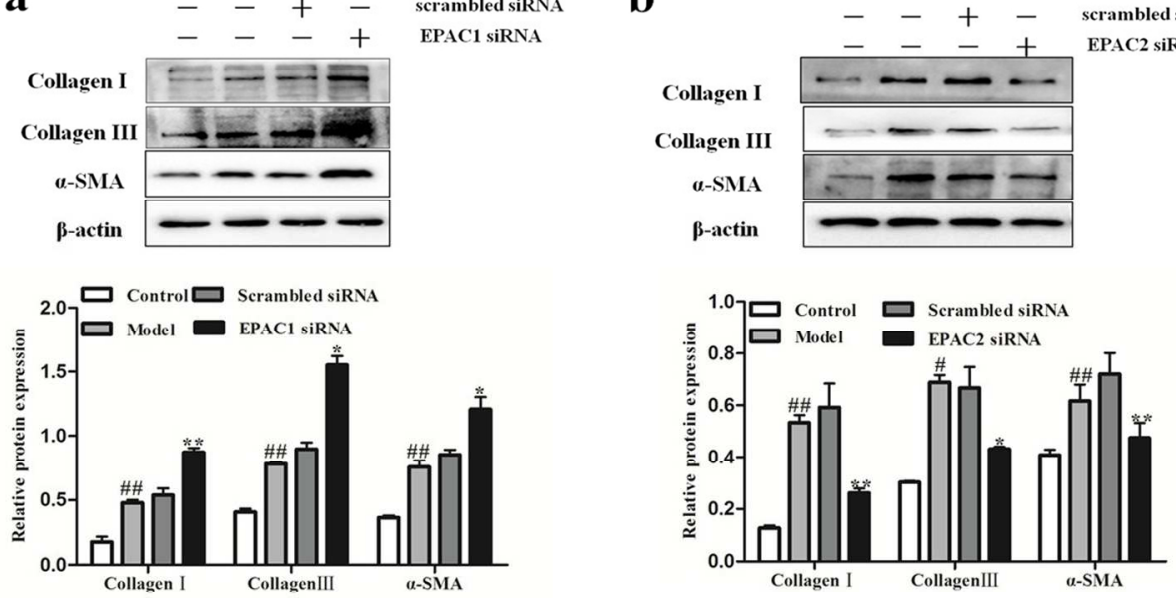

$\square$ Control $\square$ Scrambled siRNA
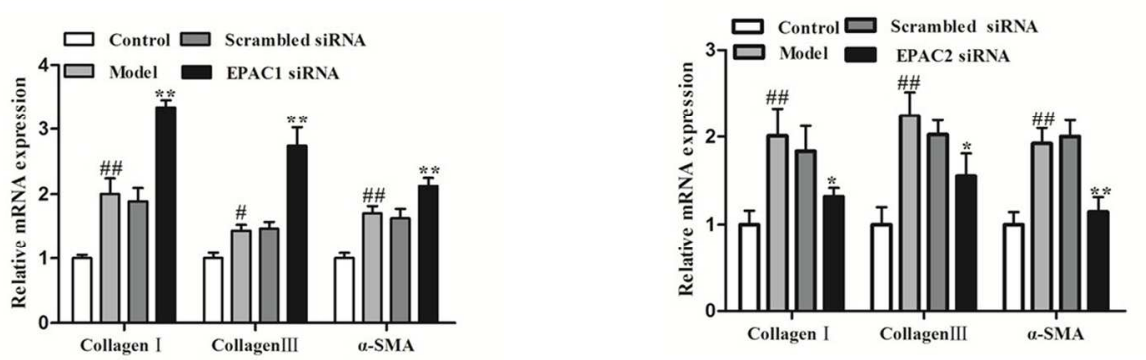

$129 \times 103 \mathrm{~mm}(300 \times 300 \mathrm{DPI})$ 
a
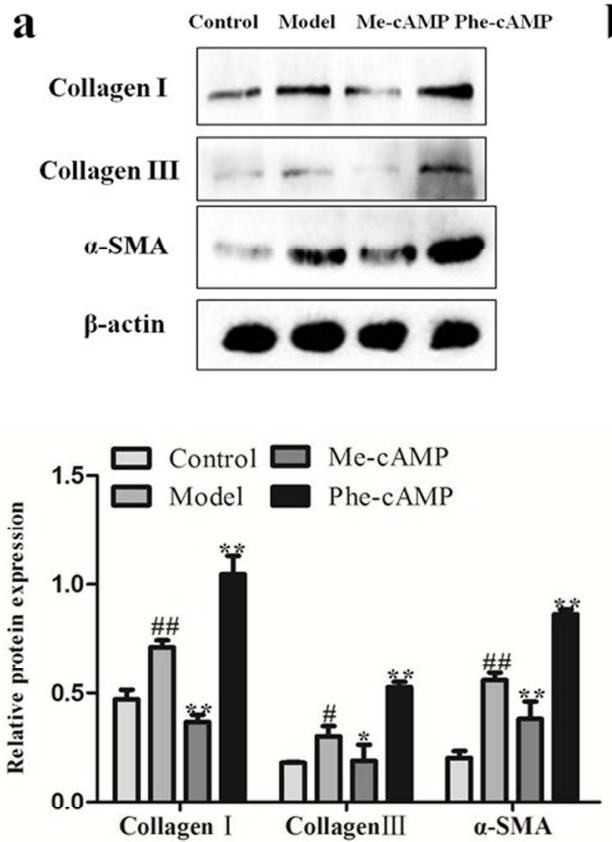

b
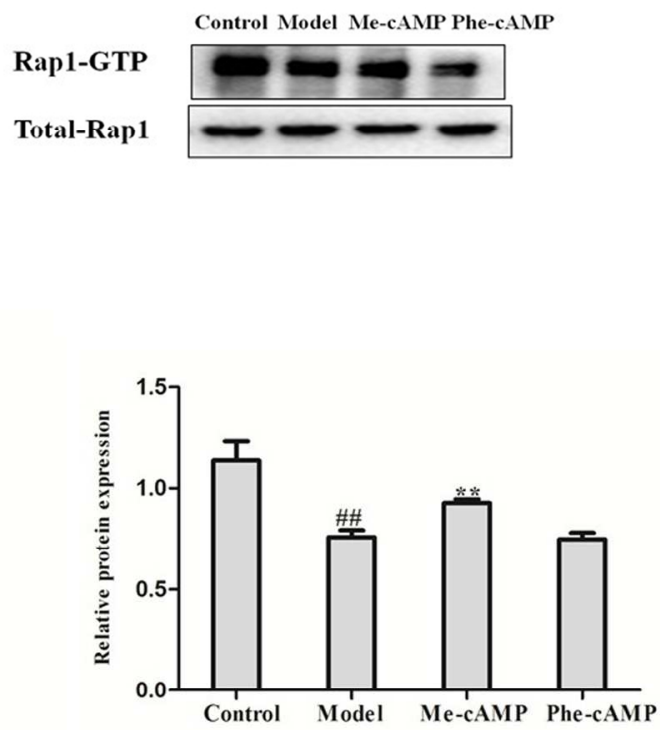

$129 \times 96 \mathrm{~mm}(300 \times 300$ DPI $)$ 